\title{
Distribution of Internet community knowledge based on traditional Communication Media
}

\author{
J. Felix Hampe, Silke Schönert, Christian Dietze and Nhiem Lu \\ Institute for IS Research, University of Koblenz-Landau, Rheinau 1, D-56075 Koblenz, Germany
}

\{hampe|schoen\}@uni-koblenz.de

Key words: ICT, Knowledge Management, Media Education, Society

\begin{abstract}
In recent years the creation of Internet-based knowledge has become increasingly significant. However, with regard to the influence and control of knowledge management processes, knowledge communities indicate that there are specific problems in creating and distributing knowledge. People without Internet access are excluded from this knowledge dissemination. Communication Centre Initiative Rheinland-Pfalz (CCIRP) - a co-operative project involving various ministries and the University of Koblenz-Landau was set up to address these problems. It has shown how concepts of knowledge generation in knowledge communities (e.g. cc-expert) can be realised and how this knowledge can be distributed using traditional media.
\end{abstract}

\section{INTRODUCTION}

Recent years have seen a dramatic increase in the amount of information stored in electronic format and available on the Internet. It has been estimated that the amount of information in the world doubles every 20 months. So-called knowledge communities are major supporters of the generation and distribution of knowledge. However, although many are able to participate in this knowledge dissemination others are left out.

Based on information supplied by the Federal Statistical Office of Germany, in $19989.4 \%$ of German households used modems for data

The original version of this chapter was revised: The copyright line was incorrect. This has been corrected. The Erratum to this chapter is available at DOI: 10.1007/978-0-387-35663-1_34 
exchange and $7.9 \%$ had Internet access. By 2000 modems were available in $15 \%$ of the households and Internet access in $17.4 \%$ of them (Statistics Bundesamt 2000).

The goal is to integrate persons without Internet access into the knowledge dissemination process by using traditional communication media like telephone or fax. This paper describes an approach for developing knowledge in a knowledge community and distributing this knowledge by heterogeneous media.

\section{KNOWLEDGE GENERATION AND KNOWLEDGE DISTRIBUTION}

Knowledge presents a state that can be attained through processes of knowledge acquisition. The acquisition of knowledge is normally demarcated as learning, which can be divided into three fundamental phases in each individual - (1) understanding, (2) storing of knowledge, (3) recollection of knowledge (Steiner 1992).

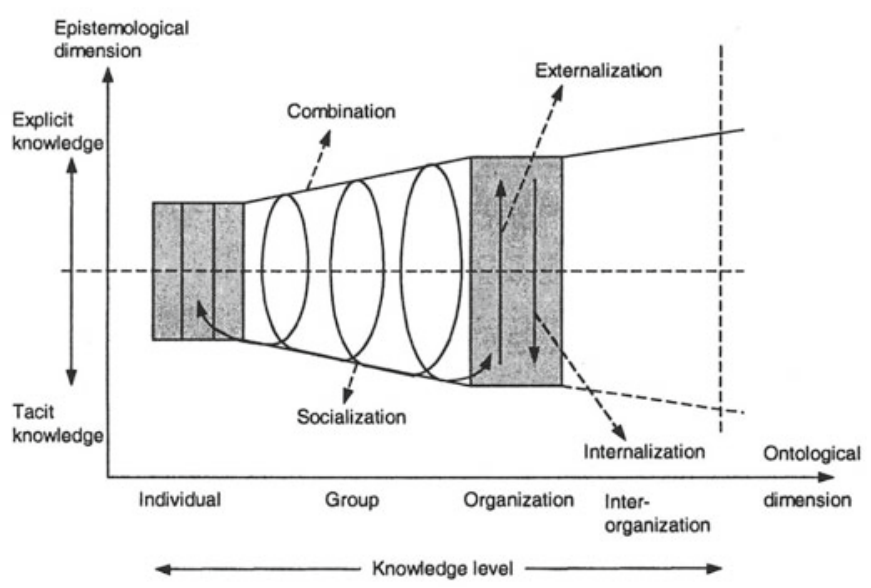

Figure 1. The spiral of knowledge

Individuals, artificially created media such as books and information and communication systems, groups, élites or whole organisations are seen as carriers of knowledge (Probst and Büchel 1994). Nonaka and Takeuchi developed the spiral of knowledge generation, which describes the process of knowledge transfer from individuals to teams and eventually the whole organisation (Figure 1). According to their model knowledge is created 
through repeated conversion activities of implicit and explicit knowledge. Implicit knowledge such as shared mental models or technical know-how is acquired non-verbally by imitating or watching experts. Explicit knowledge is available in the form of metaphors, analogies and models that are often presented verbally. The main forms of knowledge conversion are socialisation, internalisation, externalisation and combination (Nonaka and Takeuchi 1995).

\subsection{Knowledge generation in communities}

The Internet has become a so-called social medium, where people meet and communicate. People who meet frequently and engage in discussions and information exchanges based on an Internet platform are known as an Internet community or virtual community. A virtual community consists of an Internet user group who have a common interest or hobby. Virtual community members share ideas, experiences and information in the context of the specific community theme.

The information is shared in discussion forums, bulletin boards or chat systems. A community organiser can suggest discussion themes and community members create contributions or reply to contributions of other members (Shaw 2000).

On the Internet different types of communities exist (Schmidt 2000). One important type is the knowledge community. In this case knowledge is regarded as a product of communication between people. Knowledge Communities are Internet communities who's goal is to share knowledge (Schmidt 2000). All participants can offer and search for knowledge. It is important to create transparency about which knowledge exists in the community. Knowledge communities improve virtual knowledge exchange and enable the creation of so-called knowledge markets where users can participate by offering knowledge, by searching knowledge or act as a knowledge organiser - even a knowledge manager.

The intention of a knowledge community is to generate group knowledge. On the one hand this is achieved through the contributions of the members. On the other hand it is possible to activate community members by offering specific questions and discussion themes. The aggregation of a couple of contributions to a specific theme may be helpful to give a general idea of a topic. As well as the participation of interested persons it is intended that known experts participate in knowledge communities. This can be achieved by specifying expert consulting hours, creating expert discussion boards and encouraging expert contributions.

Creating a goal-oriented structure to community knowledge is a great challenge. One approach to making knowledge persistent is to build FAQ's 
and theme-specific structured documents. Another approach is to create knowledge networks. A knowledge network consists of knowledge objects and the relationships or links that exist between them. The addition of new relationships can be regarded as the creation of new knowledge. The central idea is to organise knowledge according to the concept of mind maps (Figure 2).

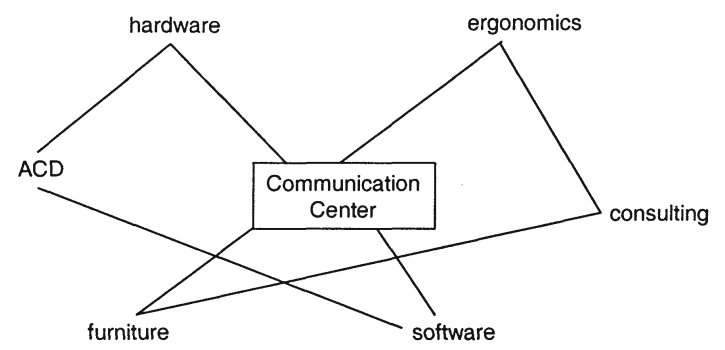

Figure 2. Example of networked knowledge

These provide a means of illustrating all relevant information for a specific topic in a graphical manner that mirrors theories about the way in which our mind works.

\subsection{Knowledge distribution depending on communication media}

There is a great amount of knowledge available on the Internet. One challenge is to offer access to this knowledge by using different traditional communication media. Experts may also be involved through this use of different media. An advanced circle of knowledge distribution is created (Figure 3).

This advanced or extended circle of knowledge distribution is determined by two aspects:

- which communication media (Table 1) is used to integrate the usergroup?

- whether the integration can be achieved with high availability $(7 * 24)$ and with low costs?

Table 1. Communication Media

\begin{tabular}{ll}
\hline Type & Communication medium \\
\hline Voice & Telephone, Mobile phone, Radio contact \\
Graphic and text & Fax, Telex, Chat, WAP, e-mail, sms \\
Voice and graphic & Internet, Conferencing systems \\
\hline
\end{tabular}




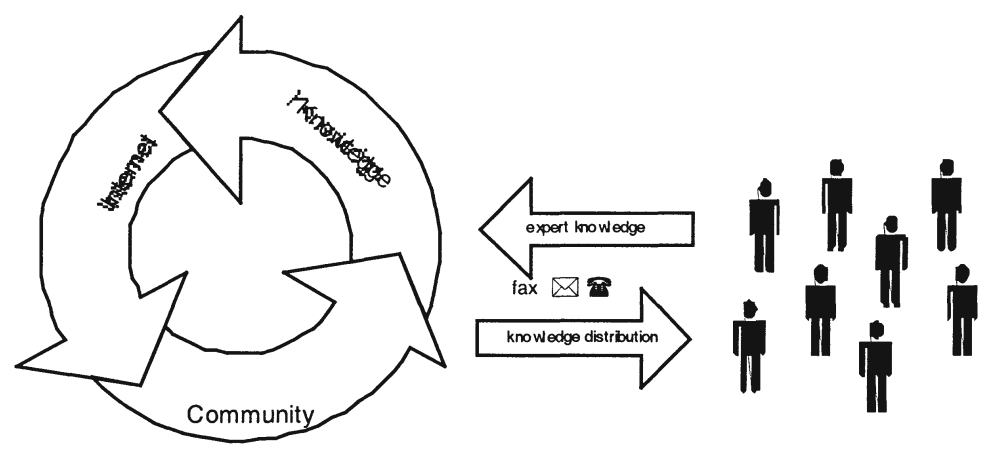

Figure 3. Extended circle of knowledge distribution in knowledge communities

Two traditional communications media are most appropriate for achieving integration of user-groups - telephone and fax. Telephone is still a favourite media for communication. The idea is to realise access to knowledge from any telephone, anywhere, at anytime. To fulfill the low cost criteria it is not appropriate to disseminate the knowledge using a person but rather by using speech technologies (Harbush et al 2001). Fax is also a favourite media. It is easy to handle, highly available and has low investment and low variable costs.

\section{CCIRP}

The Communication Center Initiative of Rheinland-Pfalz is a cooperative project between the ministry of work, social, family and health; the ministry of economy, traffic, agriculture and viticulture; and the university of Koblenz-Landau. Communication centres are call centres with a high level of media integration (Hampe and Schönert 1997).

The goal of the communication center project is to conceive an initiative to support the attractiveness of Rheinland-Pfalz for potential investors and thus create new jobs. With this in mind a project with four components was initiated. One component focuses on virtual communities. For the project a virtual community was built - known as cc-expert. 


\subsection{Virtual community}

The virtual community cc-expert is a platform where people who are interested in the communication centre can meet, share information and consult with experts frequently (Dietze 2001). It is accessed at http://www.cc-exppert.de and consists of the following functions:

- $\quad c c-n e w s$ (news about the communication centre, conferences, newsletter and cc-expert updates);

- cc-know-how (glossary, general information, communication centre locations, competitors, regulations and laws, communication centre sponsorship and communication centre technology);

- cc-personnel (their qualifications and education, comparison of wages in the communication centre, virtual employment agency);

- cc-market (offering market analysis and trends, information on sellers, consultants and education institutes);

- cc-calendar (communication centre events such as conferences, exhibitions, workshops);

- cc-contacts (contact to experts);

- cc-links.

The knowledge stored in cc-expert is organised as a knowledge network that consists of objects and the relationships between them.

The knowledge can be easily retrieved, either by actively searching through stored documents or by using certain filtering mechanisms. One of the latter is based on profiling. As a consequence, certain types of information are automatically sent to the user (push-mechanism) while others need to be actively sought by the user (pull-mechanism). The push mechanism can be effected using different communication media, for example new information objects which fit the user's profile will be sent automatically to the user (by phone or by fax). The technical platform used in the CCIRP reference lab is described in the following section.

\subsection{Reference laboratory}

In the project context a reference laboratory was designed and built at the University of Koblenz-Landau. The intention is to analyze how information can be disseminated using different types of communication media. This laboratory integrates Internet, phone and fax technologies (Figure 4). 


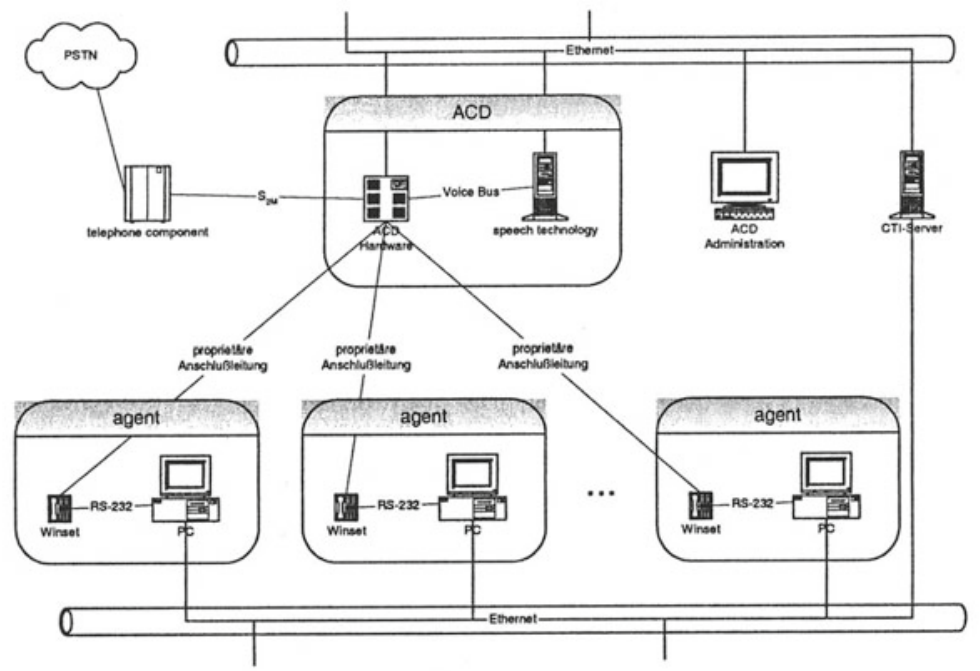

Figure 4. Structure of the Reference Laboratory

Knowledge dissemination is realized with heterogeneous communication media. These are combined in several ways. One possible scenario is that a potential investor calls the communication centre (by agent or voice system) to get information about call centre locations in the area. This investor is especially interested in financial support and contact persons. Via a speech technology based voice system he can choose between different options to:

- get an Internet address of an Internet page which contains the required information;

- get the information immediately via voice system;

- receive a fax message immediately;

- receive a letter by mail;

- get a call-back from a live agent.

After choosing the appropriate option the user can ask for further information. The system offers related information objects so the caller is able to navigate through the knowledge network.

\section{CONCLUSION}

We have presented an outline of a technology to generate and distribute knowledge. Our approach is intended to analyse how user-groups without Internet access can be integrated into knowledge communities. On the one hand the knowledge must be stored in an appropriate way, so it can be easily 
retrieved by different media, and on the other hand a comfortable platform offers different media interfaces. The first experimental uses have offered us various insights into the potential benefits. It remains to be seen, however, whether people who do not have Internet access will accept the system. Certainly the long term operation of cc-expert will lead to further requirements which arise from uses in daily life.

\section{REFERENCES}

Dietze, C., Hampe, J.F. and Schönert, S. (2001) VC3 - Konzeption und prototypische Realisierung einer Virtual Community im Communication. In Virtuelle Organisation und Neue Medien 2001, Engelien, M. and Homann, J., Josef Eul Verlag, pp. 307-327.

Harbusch, K., Knapp, M. and Laumann, C. (2001) Modelling User-Initiative in an automatic help desk system. In Proceedings of Workshop on Natural Language Processing and Neural Networks (NLPNN2001). Natural Language Processing Pacific Rim Symposium, Tokyo, Japan.

Hampe, J. F. and Schönert, S. (1997) Call Center. In Wirtschaftsinformatik 39, 2, pp. 173-176. Statistisches Bundesamt

[http://www.destatis.de/presse/deutsch/pm2000/p3400024.htm]

Schmidt, M. P. (2000) Knowledge Communities. Addison-Wesley.

Shaw, M. (2000) Handbook on electronic commerce. Springer, Berlin.

Steiner, G. (1992) Lerntheorien. In Handwörterbuch des Personalwesens, E. Gaugler and W. Weber (eds.), Poeschel, Auflage, Stuttgart.

Probst, G. J. B. and Büchel, B. S. T. (1994) Organisationales Lernen - Wettbewerbsvorteil der Zukunft. Gabler, Wiesbaden

Nonaka, I. and Takeuchi, H. (1995) The knowledge-Creating Company. Oxford University Press, Oxford, New York. 